\title{
Carnets
}

Revue électronique d'études françaises de l'APEF

Deuxième série - 5 | 2015

Imaginaires de guerre et autres conflits

\section{Léon Bocquet (1876-1954), guerre et littérature}

\section{Chantal Dhennin}

\section{(2) OpenEdition}

\section{Journals}

Édition électronique

URL : http://journals.openedition.org/carnets/438

DOI : $10.4000 /$ carnets.438

ISSN : 1646-7698

Éditeur

APEF

\section{Référence électronique}

Chantal Dhennin, «Léon Bocquet (1876-1954), guerre et littérature », Carnets [En ligne], Deuxième série - 5 | 2015, mis en ligne le 30 novembre 2015, consulté le 01 mai 2019. URL : http:// journals.openedition.org/carnets/438 ; DOI : 10.4000/carnets.438

Ce document a été généré automatiquement le 1 mai 2019.

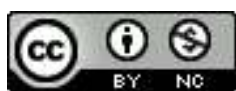

Carnets est mis à disposition selon les termes de la licence Creative Commons - Atribution - Pas d'utilisation commerciale 4.0 International. 


\title{
Léon Bocquet (1876-1954), guerre et littérature
}

\author{
Chantal Dhennin
}

1 Léon Bocquet (1876-1954) est un auteur originaire du nord de la France. Né dans une famille d'agriculteurs, il n'était guère prédisposé à la carrière de dandy qui a été la sienne au tournant du siècle. Pourtant, sa volonté de sortir de son milieu d'origine ayant été la plus forte, il est parvenu à se bâtir une carrière parisienne. Il s'est confectionné un réseau qui lui a permis de faire des études de séminariste et de pénétrer dans un univers intellectuel. Il est reçu brillamment à la licence et l'agrégation d'anglais. Il se lance alors dans le journalisme. Son itinéraire devient celui de tout homme d'esprit de son temps. Voyage en Italie, installation à Paris, cénacles littéraires (Sabatier, 1975 : 502).

2 Mais, comme le dit son panégyriste Lucien Boudet, « C'est qu'il y a eu la guerre » (Boudet, 1931). Désormais, pour Léon Bocquet, la guerre constituera un des thèmes majeurs de son œuvre, même si, curieusement, les faits de guerre ne sont guère montrés, ni décrits. Encore moins transformés en épopée romanesque. La guerre y apparaît surtout par ses représentations durant la sortie de guerre.

3 La Grande Guerre, par ce biais littéraire, est présente chez Léon Bocquet aussi bien dans les poésies (Crucifixions, Les destinées mauvaises) que dans ses romans (Le Fardeau des jours, Courages français). Cela amène à se poser la question de la Grande Guerre dans l'œuvre de Léon Bocquet, à s'interroger sur la guerre telle qu'elle fut vécue par l'auteur, et à se demander quels furent son sentiment et son comportement durant et après la guerre, et enfin quelle forme de culte de la nation, de la patrie et de l'héroïsme est véhiculée dans son livre majeur Le fardeau des jours (Paris, Albin Michel, 1924).

\section{L'écrivain Léon Bocquet pendant la Grande Guerre : son imaginaire de guerre}

4 Léon Bocquet fait partie, durant les années 1908-1909 du petit noyau envié qui, autour d'André Gide, constitue l'équipe fondatrice de la Nouvelle Revue Française. Sa sensibilité 
l'amène à prendre partie «Contre Mallarmé », et aussi contre Gide, ce qui entraîne une rupture avec le groupe initial. Désormais, le nouveau groupe ne va plus confier à Léon Bocquet que la rubrique des chroniques littéraires. Le second $n^{\circ} 1$ signe la presquerupture entre Léon Bocquet et ce cercle puissant. Ainsi, lorsque la guerre arrive, notre auteur est déjà un écrivain à l'écart du cénacle prépondérant. La guerre n'est donc pas une cassure mais plutôt la continuation d'une mise au ban plus ou moins volontaire du milieu intellectuel dominant, du monde des salons qui comptent.

\section{1) L'itinéraire de l'homme durant la guerre}

5 Léon Bocquet ne va pas faire la guerre dans l'armée d'active car il a été réformé pour déficience visuelle en 1901 (Nord, juin 2003: 7). Cette absence de participation à la vie militaire avant 1914 est importante dans le cursus de l'écrivain. En effet, rarement dans l'œuvre du romancier ou du poète, il sera question des grandes manœuvres et des soldats à l'action, maniant fusil ou grenade, avançant au pas ou en charge, vivant dans des cantonnements ou relatant un problème de relation avec un supérieur hiérarchique. La vie de garnison ou d'entraînement est largement évincée des œuvres de Léon Bocquet. Enfin, pour ne pas avoir connu les peurs des hommes du parapet, sur la ligne du front, ou pour ne pas avoir partagé l'ennui des longues heures d'attente à l'arrière ou dans les tranchées, Léon Bocquet a peu transcrit ce qui fait le quotidien de l'angoisse ou de la lassitude des combattants. La guerre de Léon Bocquet est vue par touches minimalistes, jamais avec des longs déploiements stylistiques sur un sujet que l'auteur ne souhaite pas développer (Mascarello, 1962).

6 En 1914, Léon Bocquet vit à Paris avec Marie-Thérèse Cussac, angliciste, qu'il a épousée en 1903, et avec qui il a eu deux filles, Jacqueline et Monique. Il est correcteur de langues anciennes à l'Imprimerie Nationale et cette profession lui assure suffisamment de revenus financiers pour qu'il puisse, à côté, s'adonner au plaisir d'écrire. Il a déjà, quand la guerre va commencer, publié deux recueil de poèmes qui le rendent célèbre dans son milieu: Les Cygnes noirs, en 1906, et Les Branches lourdes, en 1910, œuvre poétique pour laquelle il est couronné par l'Académie Française. Il est d'ailleurs, signe de la reconnaissance du cercle intellectuel du temps, élu vice-président de la Société des poètes. C'est donc un écrivain installé dans la capitale, vivant en famille, fréquentant le gotha des célébrités littéraires, qui voit arriver un conflit bouleversant le quotidien de ce cénacle fermé.

7 Durant la guerre, Léon Bocquet est affecté spécial, mobilisé comme secrétaire d'Étatmajor au Ministère des Régions Envahies. Cette charge lui permet à la fois de servir le pays et de rester à Paris. Ainsi, continuant à vivre en famille le soir venu, l'écrivain peut profiter de l'ambiance de la capitale en restant au plus près des préoccupations nationales grâce à la place de choix dont il dispose au Ministère. Mais, parallèlement, Léon Bocquet découvre, au jour le jour, ce que sont les souffrances des populations occupées des dix départements occupés dont il a la responsabilité dans le cadre de son activité auprès de l'État-major qui l'emploie. Ainsi, curieusement, les quatre années de la Grande Guerre sont celles d'une vie parisienne mais aussi du rapprochement progressif entre l'intellectuel dandy et la vie provinciale de sa région de naissance, le Nord envahi (Landrecies, Nord, $2003: 8$ ). 


\section{2) Un poète, un romancier qui, après un temps d'adaptation aux réalités du conflit, écrit, publie, édite}

8 Tous les individus connaissent, dès l'été 1914, une difficile phase d'adaptation à la guerre. Dans le cas qui nous intéresse, celui des écrivains et, en général, des artistes, leur sensibilité trouve des chemins nouveaux pour parvenir à s'exprimer. Certains reçoivent leur ordre de mission pour rejoindre l'armée, ils rapportent la douleur de l'expérience du combat, tel Guillaume Apollinaire, qui fut proche de Léon Bocquet (Boudet, 1931). D'autres participent à la guerre autrement, sans partir au front. C'est le cas de Léon Bocquet. On sait son affectation dans un Ministère à Paris mais comme l'auteur n'a pas décrit son ressenti face à la déclaration de guerre, à la mobilisation de ses congénères et aux premières hécatombes, on ne peut connaître son trouble face aux événements. Il est pourtant au cœur de ceux qui savent. En effet, s'il est un lieu où les premières alarmes devant les défaites du début de la guerre ont été collectées, c'est bien au sein de l'Étatmajor du Ministère des Régions Envahies. La connaissance de la déroute des armées alliées y a été analysée, et Léon Bocquet, affecté spécial en tant que secrétaire, est au milieu du dispositif. C'est probablement la raison pour laquelle il publie en 1916 avec un co-auteur Ernest Hosten une oeuvre rapportant le désastre la première année de la guerre L'agonie de Dixmude, épisodes de la Bataille de l'Yser (18-19 octobre 1914).

Le titre de cette œuvre de 268 pages, publiée à Paris chez Tallandier, est tout-à-fait en osmose avec les informations privilégiées dont Léon Bocquet dispose. Il s'agit de raconter comment Dixmude, de bourgade active et ouverte qu'elle était, est passée au statut de " ville désertée où il n'y a plus que les soldats, maisons sans toits ni pignons, fenêtres aveugles, façades branlantes, débris informes » (Léon Bocquet, 1916: 174). La préface (auteur non connu) parle de ce roman en évoquant l'œuvre d'un poète. Et il est vrai que la description de Dixmude en feu est plus une vision d'hallucination lyrique qu'un récit de faits militaires.

Sans répit, les explosions suivaient les explosions, arasant les parapets, démolissant les abris, creusant des entonnoirs, ensevelissant des hommes et projetant au loin, pêle-mêle des membres mutilés, des sacs, des armes, d'énormes mottes de glaise ensanglantée (Léon Bocquet, $1916: 145$ ).

Le préfacier insiste aussi sur «l'heureuse propagande aux idées régionalistes » que Léon Bocquet parsème dans son œuvre, constatant, par ailleurs que "l'action se trouve toujours intimement mêlée au paysage ». Il est également remarqué que l'auteur a «le souci de l'atmosphère qui donne tant d'aisance aux mouvements des groupes. » Comment comprendre le choix de Léon Bocquet de privilégier le terrain à l'action, de situer les événements en Flandre plutôt que dans la campagne des Weppes de ses origines et de raconter un combat en Belgique au lieu de le situer vers Armentières, La Bassée ou Lens, c'est-à-dire près des lieux connus de son enfance ? Cette vision de la Belgique martyre peut en effet surprendre. C'est que ce pays est celui de la seconde naissance du romancier. C'est là qu'il a rencontré ses premiers amis écrivains - Georges Rodenbach, Emile Verhaeren -, c'est là qu'il s'est exercé à dépeindre ce que son autre compagnon lettré, Francis Jammes, chef de file des régionalistes, appelle la poésie du terroir : « les ciels de ton pays, les eaux et les bois verts, et ton amour qui rit » (Léon Bocquet, 1906). L'écrivain utilise son bagage descriptif pour dépeindre une turbulence plutôt que des gains et des reculs de territoire: "Blêmes, effarés, lamentables, ça et là, des vivants pareils à des ombres évadées du tombeau, surgissaient des ruines » (Léon Bocquet, $1916: 165$ ). Telle est 
la Bataille de Dixmude vue par l'auteur: une guerre montrée du côté anthropologique davantage qu'une guerre observée du côté des stratégies et des pertes de terrain.

\section{3) Des œuvres marquées par une vision « exotique » de la guerre}

11 Léon Bocquet, après ce premier roman sur la guerre, a produit durant la guerre d'autres ouvrages et textes que l'on peut qualifier d' «exotique » eu égard aux racines terriennes de l'auteur et à son insertion dans le milieu littéraire parisien. Une préface écrite par Léon Bocquet retient spécialement l'attention, c'est l'introduction pour le roman $A u$ secours de la Serbie, Le retour d'un blessé, écrit par son ami Alcide Ramette (Ramette, 1917 : V). Il s'agit du parcours militaire d'un jeune homme du Nord, Victor Lefranc, réformé, qui, n'en pouvant plus de se sentir exclu des récits de bataille, traverse les lignes afin de s'engager pour l'Armée d'Orient. Le voilà à Marseille, à Salonique, à Krivolak, près des Portes de Fer par moins dix-sept degrés en dessous de zéro. Léon Bocquet s'empare de ce sujet pour dire que «Victor Lefranc, par un choix judicieux de touches, accède au type représentatif des hommes du Nord». En lui «s'incarnent de mâles vertus: le courage silencieux, la conscience stoïque de l'impérieux devoir et la notion idéale du sacrifice à la Patrie ». Voilà donc un récit singulier qui, par l'entremise de cette préface, accède au rang d'exemple. Cet élan patriotique exprimé par Léon Bocquet étonne d'autant plus que luimême, réformé, n'a pas fait montre d'un semblable entrain pour rejoindre les terrains de la guerre, ni sur le front occidental, ni sur les autres fronts des combats.

L'année 1918 est celle de deux courtes productions littéraires écrites par Léon Bocquet, l'une de 63 pages et l'autre de 61 pages, éditées toutes deux conjointement à Bruxelles et à Paris. La première est une œuvre à trois puisque le texte est rédigé avec Ernst Hosten (coauteur également de L'agonie de Dixmude) et qu'il est illustré par douze croquis inédits de Lucien Thomas. Le titre Un fragment de l'Epopée sénégalaise, Les Tirailleurs noirs sur l'Yser montre l'intention de l'auteur : raconter la guerre selon le biais d'une frange peu célébrée parmi les soldats qui exposent leur vie, les hommes de la Force Noire. Le début du texte confirme ce dessein: «Les pages que voici sont consacrées à l'âme obscure, loyale et fidèle d'un tirailleur sénégalais que nous avons connu. Il s'appelait Mamadou Sangho » (Bocquet, 1918: 5). Le texte, consacré au courage de ce soldat, est aussi un prétexte pour raconter l'épouvante de la boue, vue au travers des yeux d'un «exilé de sa brousse natale » qui avait « le regret des soleils évanouis ». L'angle choisi par Léon Bocquet est bien encore une interférence entre sa région natale et un ailleurs, « égaré et halluciné par l'énigme insoluble du destin ».

13 Le dernier texte écrit par Léon Bocquet durant la Grande Guerre est Villes meurtries de France, villes $d u$ Nord. Là, il n'est pas question de territoire lointain comme avec la Serbie ou le Sénégal, mais de plongée dans un passé révolu, une sorte d'exotisme du temps ancien qui prendrait pour nom nostalgie, résurrection d'antan ou mélopée de jadis. Le propos est de faire revivre une ville qui n'existe plus depuis les pilonnages allemands et les incendies de l'invasion, Lille en l'occurrence. La ville disparue dans les tourmentes de l'occupation est dépeinte comme « une force organisée, une puissance imposante, mais tyrannique et brutale » (Bocquet, 1918:6) où les riches disposent « de belles avenues sans recul » (Bocquet, $1918: 28$ ) et où les indigents habitent « des maisons noires, des maisons sans âge, penchées et cassées » (Bocquet, 1918 : 13). Car ce que décèle Léon Bocquet, dans cette description de l'atmosphère d'une ville qui n'existe plus, c'est «que les familles naissent et meurent dans le contentement de leur médiocrité » (Bocquet, 1918: 12). 
L'auteur dénonce ce défaut d'ambition d'une société - la sienne autrefois - qui se satisfait de la petitesse de son avenir. On sent, à l'extrême fin de la guerre, qu'un divorce s'est installé pour Léon Bocquet entre le sol de ses origines et un ailleurs idéalisé d'une part, et que, d'autre part le présent destructeur n'empêche pas de considérer le passé comme porteur de grande insatisfaction non comblée.

\section{Après la Grande Guerre, une absence de récit sur la guerre mais une épopée romanesque et noire sur la sortie de guerre}

Rien sur le Nord occupé de son enfance, rien sur ses condisciples en souffrance pendant la guerre elle-même, rien sur les problèmes rencontrés par les Parisiens qu'il côtoie. Léon Bocquet traverse la guerre sans le récit des grandes batailles de la Somme et de Verdun, sans le recul des soldats et sans le massif exode des évacués des dix départements occupés dont il voit cependant journellement les difficultés au Ministère où son affectation spéciale l'amène à travailler. Mais, bien qu'il faille attendre les années 1921-1924 pour que l'expulsion des non-dits enfin apparaisse, on assiste alors à un tel déferlement de noirceurs rarement teintées d'optimisme que, à coup sûr, la Grande Guerre a été un coup de poing énorme dans le psychisme de Léon Bocquet.

\section{1) Le héros-paysan}

En 1921, sort chez Payot Courages français, récits d'évasions de la Grande Guerre. Les 254 pages sont un hymne à ceux qui ont osé braver l'ennemi, les chefs, les autres. L'ouvrage est composé de six chapitres, chacun rapportant une forme de victoire personnelle sur la guerre comme si le phénomène collectif de l'occupation, de l'invasion, de la mobilisation des hommes et des esprits pouvait être vaincu par quelques désirs plus forts que le sort commun. Le premier de ces «courages français » est « un petit soldat du Nord, de la classe 1905 » (Bocquet, 1921: 15) dont le nom est Jules Duchâtelet. C'est le seul des six héros à être du terroir de Léon Bocquet puisque les lieux cités sont Salomé, Hantay, La Bassée et Marquillies, le village de naissance de l'auteur. L'histoire est simple, c'est celle des tentatives d'évasion d'un paysan retenu chaque fois dans un camp de prisonniers plus disciplinaire que les précédents après chaque ratage de sortie ou de fuite de ces lieux de détention arbitraire.

«Je ne connaissais personnellement, hier, ni Bordeux, ni Duchâtelet, ni Schönenberger. Ils m'ont été révélés par les hasards de ma documentation (Bocquet, 1921 : 11). Ailleurs, Léon Bocquet précise qu'il a rassemblé un vaste dossier et que « la matière était tellement riche et touffue que la sélection elle-même restait surabondante ». Il faut dire que "les statistiques officielles, aussi bien celles de l'adversaire que les nôtres, accusent un total de prisonniers français en Allemagne qui dépasse un demi-million» et que le nombre d'évadés peut-être fixé à 16 000. Il apparait donc que Léon Bocquet a retiré de cet amas documentaire rigoureux des feuillets qui lui ont paru significatifs de la vision qu'il voulait donner de la guerre. Son but, il le reconnaît dans la préface de son ouvrage qu'il signe luimême, est de montrer que « loin des champs de bataille où l'entraînement est contagieux, les évadés des camps d'Allemagne ont entretenu autour d'eux l'atmosphère lyrique et sacrée d'un fougueux enthousiasme, de la foi et du sacrifice » (Bocquet, $1921: 13$ ). 
Car, ce que relate Léon Bocquet, c'est le contraste entre ceux qui subissent et ceux qui luttent. D'un côté «le lamentable troupeau de captifs défilant, mornes, honteux et prostrés, par la porte de Mons » (Bocquet, 1921 : 15) ; de l'autre des hommes qui, à l'instar de Jules Duchâtelet, "tendent toute leur volonté pour ne pas s'affaler par terre" (Bocquet, 1921 : 22). Car le monde de Courages français est manichéen : les tortionnaires allemands avec ceux qui plient la tête devant eux, pour le camp négatif; les paysans septentrionaux à l'endurance butée et à la ténacité têtue, pour le camp positif. Il faut retirer de cette vision duale l'importance du concept de "paysans septentrionaux » qui s'impose dans l'esprit de Léon Bocquet. Pourtant Duchâtelet, le héros aux douze évasions, n'est en rien un homme de la terre ; il est, dès le début de l'histoire, artisan boulanger auprès de son oncle; ensuite, il est qualifié de mitron; on le voit devenir houilleur en train de ramper dans les galeries basses pour abattre le charbon; il est employé enfin comme menuisier : « la menuiserie, ça me connaît. J'ai cela dans le sang. Mon grand-père était menuisier. Mon père aussi » (Bocquet, 1921: 23). Mais, en dépit de ces métiers d'artisans et d'ouvriers, le protagoniste de ce récit est un paysan, un héros-paysan, puisque c'est là le titre du chapitre, comme si la terre du Nord que Léon Bocquet a quitté a fui - apportait cependant la noblesse des actes davantage que les autres professions.

\section{2) La réhabilitation des Destinées mauvaises}

Léon Bocquet sort en 1923 un ouvrage qui semble déconnecté par rapport à son temps, Les destinées mauvaises, édité à Amiens chez Edgar Malfère. Nulle allusion à la Grande Guerre qui pourtant est l'objet des écritures du moment. Non, ici, il s'agit d'un livre de compassion évoquant cinq auteurs injustement oubliés par l'histoire littéraire. L'ordre est chronologique : Moreau se suicide en 1838 ; Corbière décède de privations en 1875 ; Pierre de Querlon meurt en 1904, après deux mois de maladie ; Guy de Villartay s'éteint en 1907 à Paramé ; Léon Deubel se donne la mort en 1913, ne supportant plus la pauvreté. Ces cinq poètes sont considérés comme ayant eu des «destinées mauvaises», des parcours malheureux.

19 Léon Bocquet, et c'est en cela que cet ouvrage se rapproche de Courages français que vient d'écrire précédemment l'écrivain, considère que ces auteurs ont été accablés et désenchantés mais que, le plus souvent, ils ont été eux-mêmes à l'origine de leurs souffrances et de leurs misères. C'est, par opposition, la valorisation du mythe du hérospaysan qui tend toute sa volonté " pour ne pas s'affaler par terre». Destinées mauvaises argumente en montrant que, pour le moins, les comportements des cinq infortunés ont contribué à développer les déboires déjà survenus dans leurs vies. A la différence des Poètes maudits de Verlaine, qui d'ailleurs commencent par Tristan Corbière et se terminent par Verlaine lui-même, Léon Bocquet nie l'acharnement complet de la fatalité et l'indifférence totale de la société. Il explique que Pierre de Querlon a connu des excès de travail et de plaisirs, que Corbière a nargué la mort, que Moreau, à force d'être cynique, a perdu toute retenue, que Deubel s'est complu à paraître maudit et miséreux et que Villartay n'a guère cherché à sortir de son cercle avant sa mort prématurée ${ }^{1}$. Ainsi, pour Léon Bocquet, les tourments psychologiques, les misères matérielles et l'incompréhension du public sont des entraves au plaisir de vivre et d'écrire mais qu'il faut parvenir à dominer.

Le contexte de la sortie de guerre vient donc réveiller cette idée que la fatalité peut être exorcisée grâce à une attitude résolue. Léon Bocquet s'y emploie pour ces poètes et ces 
amis disparus trop tôt : il incite les lecteurs à recueillir et à relire les textes oubliés; il évoque l'admirable langue utilisée par ces écrivains, faite de verve et de nervosité; il montre la sincérité et l'authenticité de leurs textes; il invite non pas à admirer mais à aimer leurs œuvres. Cette réhabilitation étonnante des cinq poètes aux destinées mauvaises laisse supposer que Léon Bocquet, lui-même, en redonnant vie à des créateurs presque oubliés, travaille par altruisme, bien sûr, mais aussi prêche pour son propre parcours qu'il aimerait inscrire également dans la haute lignée française des écrivains de renommée. En effet, de même que ces auteurs d'avant-guerre tombent quasiment dans l'oubli, lui se voit confronté à l'après-guerre caractérisée par son exclusion progressive du cercle de la $N R F$, la mort d'amis chers, l'étroitesse inquiétante de son réseau parisien, et surtout l'éloignement des auteurs régionalistes qui rejoignent leurs ports d'attache en province. Au moment qui voit la naissance des avant-gardes poétiques avec le Manifeste du surréalisme en 1924, Léon Bocquet tente de rester visible en se créant une carapace volontariste.

\section{3) La guerre versus poésie}

Lorsque Léon Bocquet donne son recueil de poèmes Flandre à éditer à la Maison des Poètes à Paris en 1901, il est satisfait du résultat. Pourtant, la guerre venue, il reprend ses quarante-cinq sonnets, les retravaille et les réorganise pour proposer une réédition sous le titre Evocations de Flandre en 1927 et, après encore bien des avatars, il annexe ces poésies, encore une fois transformées, dans Ciguës en 1938. L'étude de ces transformations est donc significative afin de voir l'influence de la guerre sur le contenu et les métamorphoses poétiques de l'œuvre de cet auteur. Le recueil d'origine, Flandre, est composé d'un mouvement descendant - les premiers poèmes s'appellent Désolation puis Abandon - mais l'ensemble se termine par une phase optimiste dont le titre Renaissance, qui clôt le sujet, est à lui seul tout un programme : « Ton exil a cessé, va, sois fière et forte / Autour de toi s'élève un triomphe de cris. » (Bocquet, 1901).

La version remaniée présente en effet d'importantes différences, et spécialement l'inclusion de termes locaux. Absents dans les premières éditions, voici que les mots de la langue quotidienne du Nord, à présent, viennent en force. Le poème Villes mortes, réécriture de Vieilles villes, est un bon exemple pour observer ces changements : au lieu et place de "quartiers étroits ", l'auteur a écrit « dans le minck à l'étroit» (Bocquet, 1927). Ce détail, bien qu'il n'apparaisse pas révolutionnaire, traduit le changement des représentations de Léon Bocquet. Alors que l'écrivain, avant la guerre, se plaisait à faire du régionalisme non teinté de localisations précises afin que les horizons qu'il décrivait puissent convenir à tous les francophones, le voici maintenant qui s'inscrit dans un endroit et dans une histoire. Le simple fait de placer le mot " minck», terme signifiant marché aux poissons en langue flamande, implique que le poème trouve sa place dans un terroir spécifique. Léon Bocquet, qui avait jusque-là tenu à distance les villages de sa région d'origine afin de faire apparaître l'entière humanité et non un pan spécifique, fait un retour sur lui-même et inclut désormais des éléments de pittoresque biographique. L'homme de lettres parisien est maintenant plus à l'aise avec son héritage culturel. La guerre est passée par là.

Le rôle de la guerre est primordial pour comprendre l'enracinement progressif de Léon Bocquet dans sa région natale. Le Nord fait partie des dix départements envahis et occupés, certes; mais les méfaits de la guerre ne s'arrêtent pas là. Le secteur 
géographique de Marquillies, village natal de l'auteur, est situé sur l'arrière-front occidental, en lisière de la zone rouge détruite à $100 \%$ par les bombardements alliés et allemands. Rien n'est reconnaissable des maisons, des paysages, des horizons familiers de l'auteur. Rien ne subsiste des petits ateliers ni des appentis qui faisaient des niches secrètes pour qui aimait à se perdre dans les méandres des rues. La population, évacuée, n'est revenue qu'à $65 \%$; ce sont des étrangers au canton qui s'occupent de la reconstruction, qui habitent les maisons neuves, qui recomposent à leur avantage le tissu social d'antan. Il est temps que cette région disparue renaisse, au moins en poésie. Voici apparaître «bélandre » au lieu de " caraque » pour les bateaux remontant les canaux, et "béguine» au lieu de "nonne». Voici que les termes picards de courtils (=jardins), de ducasse (=fête locale) et d'escarbilles (=résidu de charbon) font leur apparition. Voici que la région du sud-ouest de Lille s'affirme et ose dire ses mots, elle qui souffre de la méconnaissance de son martyre de la Grande Guerre.

\section{La hantise de la violence chez les survivants de la guerre : Le fardeau des jours}

Le point de vue de Léon Bocquet sur la Grande Guerre est très pessimiste. Son roman Le fardeau des jours (Bocquet, 1924), édité chez Albin Michel, en est le témoignage. Tous les thèmes traités convergent vers cette idée que la guerre a été davantage une profonde dévastation intérieure qu'une dévastation des lieux, pourtant irrémédiablement détruits.

\section{1) L'imaginaire épique au service d'une description pessimiste des retours d'évacuation dans la zone rouge du front français occidental}

Le roman situe l'action en 1918-1919, lors du retour d'évacuation des réfugiés qui découvrent l'apocalypse avec leurs maisons ruinées et leur environnement défiguré. Ce retour est perçu comme un passage, un moment transitionnel entre le monde détruit et celui à venir. «Ce passage, tel un étau, enserre et broie ses victimes » (Hache, Nord, 2003 : 23). La confrontation avec cette vision de catastrophe est annoncée par la citation de l'Evangile mise en exergue : «Prends ta croix, et va ». Elle se concrétise dès les premiers chapitres par la véritable montée au Golgotha qui est l'avancée progressive vers les cercles d'enfer que sont devenus les villages démantelés par les flammes et rasés par les obus. «La gare obstruait de ses débris l'ouest de la ville, entassant dans un chaos de bouleversement des wagons culbutés ou fracassés avec les briques pilées des maisons riveraines anéanties» (Bocquet, 1924: 15). L'auteur décrit et dénonce. Il explique les dépravations afin de susciter de l'empathie pour sa terre natale, ici nommée, ici localisée, ici assumée géographiquement. Le propos est en effet, au moins en partie, de crier le désarroi des populations qui reviennent et sont privées de tout. Et, lorsque les habitants revenus reçoivent une aide publique, c'est un jour un baraquement, une autre fois un lot de poulets, eux qui, hier fiers propriétaires de domaines prospères ou de boutiques florissantes, en sont réduits à quémander auprès d'élus qui veillent à leur trace électorale plutôt qu'au bien collectif. Lourde charge, que ce roman. Comme si Léon Bocquet avait des comptes à régler avec le Ministère des régions envahies où il a été employé durant la guerre, et qui le nourrit encore à l'heure d'écrire cet ouvrage accusateur. 
'épique est à chaque page. Il se trouve dans l'amplification avec des termes qui créent de l'intensité et de l'emphase : " Angèle et Zoé mordirent à dents longues et rechignantes le pain grisâtre qui avait le goût de l'air humide et une odeur de rance " (Bocquet, 1924 : 16). Il apparaît dans des procédés d'exagération que Léon Bocquet utilise avec abondance. Répétition : «Rien. Ni tours, ni toitures. Mais la plaine, la plaine désespérément morne et unie avec cette gangrène rongeant la glaise, la plaine entaillée par l'estafilade à perte de vue de la route suivie » (Bocquet, 1924: 21). Gradation : «C'étaient d'abord des abris de béton, lourds cubes aveugles et sournois. (...) Ensuite, dans quelques rangées d'arbres, des postes d'observation juchés dans les coupets de fourche. Ou bien des abris de tôle ondulée bombant le dos " (Bocquet, $1924: 22)$. Hyperboles: "Des banalités s'échangent entre capuches. Les cloches noires ondulent et se déplacent. Elles chuchotent » (Bocquet, 1924 : 316). Il va jusqu'au merveilleux : " Tout-à-coup, une voix pointue s'éleva, claire, joyeuse, allègre, et qui chantait » (Bocquet, 1924: 320). Ces procédés, se cumulant l'un l'autre, forment des redondances qui assiègent le lecteur et lui font vivre en symbiose les actions des protagonistes.

Le champ lexical utilisé, comme l'indique le titre, est celui du fardeau. L'exemple de la page 39 montre à quel point les mots sont en rapport avec cette idée de charge qui fait ployer les êtres et les choses. Les êtres sont accablés : «Lourdement, Mélie gagna un coin libre où elle s'éboula généreusement sur les voisins ». Le climat est pesant : « Il n'y eut pas une parole échangée entre la mère et la fille. Elles ne levèrent pas la tête, ne s'épièrent pas du regard. Elles s'ignoraient, voilà tout. " Tout se délite, comme si un écrasement généralisé ne laissait partout que des restes et des résidus : «Bientôt, l'écroulement doux des bûches calcinées et l'égouttement de l'eau qui tombait quelque part du toit crevé (...) hantèrent les intervalles d'accalmie de la bourrasque et les sifflements monotones des respirations. » L'idée de ces charges qui s'accumulent au propre et au figuré créent une ambiance de menace constante où la peur le dispute à la gravité pour faire une sorte de caricature de sac trop lourd à porter par chacun. Le Nord durant la sortie de guerre, selon Léon Bocquet, est vraiment dans le registre du fardeau.

\section{2) La dénonciation d'une dévastation intime des réfugiés}

Les hommes du Fardeau des jours sont marqués par l' "épreuve du désastre " (Brossat, 1996) qui, plus que la ruine des territoires et des maisons, est la dévastation intime, ce que Hannah Arendt a nommé "l'échec du dire après la Première Guerre mondiale " (Arendt, 1973). Personne, parmi les personnages du roman ne parvient à communiquer avec l'autre. Tous ressassent leur dégoût d'avoir été chassés par l'occupant, d'avoir été "parqués comme vil bétail ou pourceaux à l'encan, là où il avait plu à l'ennemi de refouler les gens de la ligne de feu » (Bocquet, 1924: 44), mais c'est dans le quant-à-soi que se déverse cette bile amère. Voilà, en effet, à quoi a abouti la guerre : à générer une incommunicabilité des esprits et des intelligences. Dès la découverte des granges et des remises rasées, avec le vent qui entre et sort par les ouvertures des fenêtres et des portes démolies, le silence s'est abattu. "Les uns et les autres se sentirent tout-à-coup étrangement las et fourbus. La tristesse ambiante entrait en eux, à mesure qu'ils avançaient. Ils ne parlaient plus » (Bocquet, 1924 : 44). La fermeture à la parole d'autrui et son corollaire le mutisme sur soi sont les deux grandes caractéristiques de la nouvelle solitude individuelle issue de la guerre selon Léon Bocquet. 

Vasseur, aurait pu se dissoudre dans des habitudes ancestrales retrouvées, dans des traditions séculaires d'entraide, dans des rites réparateurs. Rien de tout cela. La solidité intergénérationnelle est ébranlée, à l'instar du désert d'affection entre mère et fille que l'auteur dépeint avec une aigreur sans pareille : «A quelques pas l'une de l'autre, ces deux femmes semblaient séparées par des siècles » (Bocquet, 1924: 58). Les habitudes du soutien familial sont également comme perdues à l'intérieur de ce clan qui n'est plus soudé que par la marche en avant et la recherche d'un toit où s'arrêter : «Sur la solitude navrée, sur le village mort, sur les cœurs en détresse, s'appesantissait du silence, du silence, du silence» (Bocquet, 1924: 62). Il reste enfin, au nombre des marqueurs réparateurs, les églises et les assistances publiques. Même ces liturgies-là sont inefficaces tant les affrontements intimes sont profonds. Le prêtre du Fardeau des jours de Léon Bocquet, mais aussi le maire et également le député, ne sont pas précisément des hommes magnanimes, généreux et indulgents. "En surplis raide qui donnait le frisson, le curé, exact et militaire, s'amena, marchant de son allure de fantassin conquérant, sur la glace qu'écrasaient ses lourds souliers » (Bocquet, 1924: 319). La description de l'abbé Pastoureau installe l'impossibilité de l'écoute régénératrice.

qui apportent une autre voix sont le rebouteux, l'infirme et l'anarchiste. Le rebouteux, appelé le vieux Marche-sans-Cesse ou bien encore Doudou le Guérisseur, amène au moins un regard et un contact, éléments qui manquent le plus dans l'individualisme de la sortie de guerre: "Ses longues mains à l'onction sacerdotale, ses mains levées sur des affres ou des remords, savaient, ainsi que celles d'un dieu, provoquer des prodigues. (...) Il murmurait des invocations, (...) il récitait des prières latines " (Bocquet, 1924 : 220-222). L'infirme a pour nom Narcisse. Il est le seul à s'intéresser à Mélanie : "Ce n'est pas un fameux parti que je vous offre. (...) Mais quoi! Il y a tant d'estropiés aujourd'hui, depuis la guerre, qu'on prête moins d'attention à ces choses-là. Mes bras sont valides, allez» (Bocquet, 1924 : 144). L'anarchiste s'appelle Anthyme; il amène la possibilité d'une autre vie grâce aux discours utopiques dont il abreuve les hommes du village : « Il avait lu pêle-mêle et mal digéré des poètes, des romanciers, des théoriciens de la cité nouvelle. Il s'y était gavé de thèses séditieuses et de rêveries ultrahumanitaires. Il ne jurait plus que par Zola, Hugo, Karl Marx, ces prophètes » (Bocquet, 1924 : 103). Mais le rebouteux, l'infirme et l'anarchiste, porteurs de mort chacun à leur façon, ne sont pas non plus les sauveurs attendus.

\section{3) Le Fardeau des jours : un condensé des désordres nés de la guerre}

31 C'est qu'un phénomène implacable est en place, installé par Léon Bocquet dès le début du Fardeau des jours, distillé au fil des pages, et accablant pour les protagonistes jusqu'à quelques pages de la fin du roman, le phénomène du bouc émissaire. Des figures d'acteurs-bourreaux et de persécuteurs montrent le résultat psychologique effrayant des quatre années de guerre. Le premier des désordres est la recherche d'un coupable au sein d'une communauté qui ne supporte plus son déclassement social. Le village de Marquillies où est né Léon Bocquet, symboliquement désigné ici sous le toponyme de Willy, était au début du xxe siècle, une commune dynamique. La bourgeoisie locale y avait installé tôt ses premières machines à vapeur et, de métiers à fabriquer des pantoufles aux fabriques de sucre, de mélasse et de chicorée, tout le secteur bruissait du travail de ses habitants. Certains, ouvriers à la mine de Lens, d'autres, tenanciers des estaminets et des auberges, 
vivaient bien grâce aux allées et venues de tout ce canton florissant. La guerre a tout anéanti et la relance tarde. À qui en revient la faute?

La désignation de la victime expiatoire est en route car il faut extraire l'intrus qui empêche le groupe, le quartier, le village de redémarrer. La personne qui dérange, qui est hors des normes constitutives de la communauté, qui menace la cohésion de l'ensemble, doit être signalée afin d'être expurgée. Dès le début du roman (Bocquet, 1924: 10), Mélanie est montrée comme étant celle qui, différente, apporte le désordre autour d'elle. Premier signe distinctif : elle est trop belle, "tentation et damnation des hommes». Second critère de désignation : « elle avait lancé son dragon hors du jeu, elle avait forfait à l'honneur, elle s'était fait remarqué, comme on disait. » Enfin, argument suprême, elle avait fauté avec un Allemand: "À moins de vingt ans, pensez donc! Et quand? Aux premières semaines de l'occupation ennemie, alors que les soldats français étaient fauchés comme des moissons rouges. » La simple jalousie commence à prendre des allures d'holocauste. La violence victimaire est engagée.

Léon Bocquet installe une polarisation sur Mélanie, individu unique à voir ainsi converger vers elle les haines auparavant éparpillées. Violence unilatérale et unanime (Girard, 1990 : 122) contre celle "qui avait récolté l'ivraie au lieu du probe froment " (Bocquet, 1924 : 11). Etape un : L'exclusion alimentaire. La part de pain qui revient à Mélanie et à sa fille Maria est plus réduite que celle distribuée aux autres membres de la famille. Etape deux : L'exclusion physique. La chambre affectée à la mère fautive et à l'enfant de la faute est un réduit désaffecté. "Des étrangers seraient mieux traités " (Bocquet, 1924 : 110). Etape trois : L'exclusion psychique : Mélanie, faute d'être acceptée et écoutée, glisse peu à peu vers la folie ordinaire. « Elle passait des journées entières à se démener, à marmonner des propos incohérents ou à chantonner d'une voix étrange » (Bocquet, 1924 : 287). Mais, au prix du glissement progressif vers le néant de Mélanie, voilà que des mains se tendent vers la petite Maria, que "la lente reconstruction" (Bocquet, 1924: 306) du secteur apparaît. Le livre se termine donc, en un chapitre final qui tranche avec la tonalité morose et noire du roman, sur une triple exclusion fondatrice. Fondatrice d'optimisme. En réalité, le «fardeau des jours» se poursuit longtemps encore durant l'après-guerre, pendant près d'une quinzaine d'années aussi le pays de Léon Bocquet ne parvient pas à retrouver rapidement la dynamique qui était la sienne au début du $\mathrm{xx}^{\mathrm{e}}$ siècle.

\section{Bilan}

Littérature mi-épopée, mi-témoignage, les œuvres de Léon Bocquet, parues durant la guerre et la sortie de guerre, sont incontestablement le fruit de la période.

Marqué dans ses racines, bien que parisien d'adoption, l'écrivain du Nord a souffert de la dévastation de sa terre natale. Un glissement s'est effectué chez l'écrivain, depuis la suggestion intime du refus de la guerre jusqu'au besoin du bouc émissaire afin d'exorciser son mal-être de voir tant souffrir les villages de son enfance. Trois étapes sont à observer. La première traite de la guerre par des voies détournées, presque " exotiques ", c'est la guerre chez les autres, par les autres, les Sénégalais, les Serbes, les Belges. La seconde prend des chemins poétiques, remaniés après le conflit, pour traduire, avec des accents régionalistes, le martyre de la région natale de l'auteur. Et enfin, dans le cas du Fardeau des jours, il apparait incontestable que l'homme qui fit la guerre à l'abri des bureaux parisiens est si marqué par les dévastations matérielles, physiques et mentales de la 
guerre qu'il façonne un roman spécialement centré sur le mal de vivre des populations évacuées, celles de ses terres d'origine.

La forte prégnance imaginaire de l'auteur est associée à la dénonciation des notions de patriotisme et même de foi. Elle critique une guerre qui a cassé les hommes en revisitant le mythe des processus victimaires. Elle fait voir grâce au feu d'artifice des mots, utilisés ici dans un registre noir et pesant, les innombrables dégradations au corps et au cœur des régions et des individus. On aura compris que c'est sur le terrain de l'anthropologie et de la mémoire culturelle que se fonde l'œuvre de Léon Bocquet.

\section{BIBLIOGRAPHIE}

BOCQUET, Léon (1906). Les Cygnes noirs. Paris : Mercure de France.

BOCQUET, Léon (1910). Les Branches lourdes. Paris : Beffroi.

BOCQUET, Léon (1916). L'agonie de Dixmude, épisodes de la Bataille de l'Yser (18-19 octobre 1914). Paris :

Tallandier.

BOCQUET, Léon (1918). Un fragment de l'Epopée sénégalaise, Les Tirailleurs noirs sur l'Yser. Paris :

Librairie Nationale d'Art et d'Histoire, Bruxelles : Van Oest.

BOCQUET, Léon (1918). Villes meurtries de France, villes du Nord. Paris : Librairie Nationale d'Art et d'Histoire, Bruxelles : Van Oest.

BOCQUET, Léon (1921). Courages français. Paris : Payot.

BOCQUET, Léon (1924). Le fardeau des jours. Paris : Albin Michel.

BOCQUET, Léon (1923). Les destinées mauvaises. Amiens : Edgar Malfère.

BOCQUET, Léon (1929). Crucifixions. Paris : Messein.

BOUDET, Lucien (1931). Léon Bocquet, Curieux Homme. Paris : Messein.

BROSSAT, Alain (1996). L'épreuve du désastre, Le XXe siècle et les camps. Paris : Albin Michel.

GIRARD, René (1982). Le Bouc émissaire. Paris : Grasset.

LANDRECIES, Jacques (dir.) (2003). Nord, Numéro spécial : Léon Bocquet, $\mathrm{n}^{\circ} 41$.

MASCARELlo, Anna (1962). La revue 'Le Beffroi' de Léon Bocquet. Saarbrücken : [s.n.].

RAMETTE, Alcide (1917). Au secours de la Serbie, Le retour d'un blessé. Paris : Plon.

SABATIER, Robert, (1975). « Poésie aux cent visages - Poètes sociaux - Léon Bocquet (1876-1954) », Histoire de la poésie française, vol. 5.

VERLAINE, Paul, (1884). Poètes maudits. Paris : Léon Vanier. 


\section{NOTES}

1. Paul Renard, Nord, $n^{\circ} 41$, juin 2003, Spécial Léon Bocquet.

\section{RÉSUMÉS}

Pour Léon Bocquet (1876-1954), la guerre constitue un thème majeur de son œuvre. Mais, curieusement, les faits de guerre eux-mêmes n'y sont ni montrés, ni décrits. Encore moins transformés en épopée romanesque. La guerre y apparaît surtout par ses représentations durant la sortie de guerre, les transformations qu'elle implique, sa réfutation au nom de l'idéal pacifiste et des cruautés qu'elle entraine. La Grande Guerre, par ce biais littéraire, est présente chez Léon Bocquet aussi bien dans les poésies (Crucifixions, Les destinées mauvaises) que dans ses romans (Le Fardeau des jours, Courages français). La question de la représentation de la Grande Guerre dans l'œuvre de Léon Bocquet étant corrélative d'abord à celle de la participation de l'homme dans la littérature, dans la guerre et dans la littérature de guerre, ensuite à la fidélité au récit combattant et enfin au culte de l'idéal héroïque, on se demandera quelle fut la guerre de Léon Bocquet, quels furent son sentiment et son comportement durant et après la guerre, et enfin on verra quelle forme de culte de la nation, de la patrie et de l'héroïsme est véhiculé dans son livre majeur Le fardeau des jours (Paris, Albin Michel, 1924).

For Léon Boucquet (1876-1954), war is a major subject of his works. Curiously, War's events aren't shown nor described in it. Nor are they transformed into a romantic or epic poem. War is present mainly through its representation at the end of the war, the transformations it implied, le refusal of an ideal of pacifism and the cruelties it entailed. WWI is literarily present in his works through Poems (Crucifixions, Les destinées mauvaises) and in Novels (Le Fardeau des jours, Courages français). The question of the representation of WWI in Léon Boucquet's work is linked to that of the participation of man in literature, in war and in war literature. We will ask ourselves how was war for Léon Boucquet, which were his feelings and his behavior during and after the war, as well as what worship of heroism and of the nation is conveyed in his chief novel Le fardeau des jours (Paris, Albin Michel, 1924).

\section{INDEX}

Mots-clés : récit de guerre, imaginaire épique, trauma, héroïsme, littérature

Keywords : talk about battles, epic imaginary, engagement and pains, heroic posture, literature

\section{AUTEUR}

\section{CHANTAL DHENNIN}

Université Lille Nord de France

chantal@dhennin.com 